\title{
Effect of Continuous and Reciprocating File Motion on Microcrack Formation in Root Canals During Retreatment: A SEM Study
}

\author{
Aishwarya Singh ${ }^{1 *}$ and Rahul Maria ${ }^{2}$ \\ ${ }^{1}$ Post Graduate Student, Department of Conservative Dentistry and Endodontics, \\ Bhabha College of Dental Sciences, India \\ ${ }^{2}$ Professor and HOD, Department of Conservative Dentistry and Endodontics, \\ Bhabha College of Dental Sciences, India \\ *Corresponding Author: Aishwarya Singh, Post Graduate Student, Bhabha College \\ of Dental Sciences, Bhopal, Madhyapradesh, India.
}

Received: February 07, 2022

Published: February 17, 2022

(C) All rights are reserved by Aishwarya Singh and Rahul Maria.

\begin{abstract}
Aim: The aim of this study is to compare microcrack formation and propagation in apical one third of root after retreatment, while using two different retreatment files.

Material and Methodology: A total of 50 human mandibular premolar teeth with straight root canals were selected and coronal third enlargement done with one flare. The biomechanical preparation of the teeth were done using Protaper Next rotary files X1 and X2. Obturated using gutta-percha with AH Plus sealer by lateral condensation technique. The samples were divided into two retreatment groups -Group I- Protaper Universal Retreatment file system and Group II-Wave One Gold primary File. Then microcracks were assessed using scanning electron microscope. The data were statistically analysed using Pearson Chi square test, Odd's ratio and Relative risk/Risk ratio.

Results: After retreatment, microcracks are present in both the groups. The PTUR system caused significantly more microcracks than the Wave one gold file system in the apical one third of root.

Conclusion: Within the limitations of present study, continuous motion file group produced significantly more percentage of incidence and propagation of dentinal microcracks as compared to reciprocation motion file group.
\end{abstract}

Keywords: Apical Microcracks; Continuous; Protaper Universal Retreatment File; Reciprocation; Retreatment; Wave One Gold File

\section{Abbreviations}

NiTi: Nickel Titanium; PTUR: Protaper Universal Retreatment File; SEM: Scanning Electron Microscope; VRF: Vertical Root Fracture

\section{Introduction}

Biomechanical preparation of the root canal is the major step of root canal preparation as it will give more area for the irrigants and intracanal medicaments to adequately remove bacteria and their byproducts [1].
Root canal treatment failure may occur because of the continual microorganisms or resurgence of microorganisms in canal through apical or coronal pathway. A successful retreatment procedure can be accomplished after removing the old root canal filling material and then completing the cleaning, preparation and obturation [2].

If initial root canal treatment fails, then the first line of treatment is nonsurgical endodontic retreatment, it aims to reduce the microbial load and it needs complete removal of the root canal filling material for proper cleaning and obturation of the canal [1]. 
For removing filling material from root canal system different methods are given like by using hand files, solvent, heat ultrasonic instruments, NiTi rotary instruments [1].

Now-a-days, the NiTi rotary files are in demand due to the superior properties than other stainless steel files. Hence, NiTi rotary files are extensively used in retreatment procedures and biomechanical preparation [2].

During retreatment procedures, the apical third has more amount of residual filling materials due to the more anatomic variations, hence, more stresses are faced during cleaning of the canal. So, more time is required which increases the probability of microcrack [1].

Nevertheless, factors other than the endodontic files in retreatment; force of instrumentation and obturation, high concentration of sodium hypochlorite, post preparation, tooth anatomy, etc. are also considered as the factors for the dentinal defects (microcracks) formation $[3,4]$.

For better removal of root canal filling material after retreatment instruments, more instrumentation with increasing instrument sizes used, which further increases the probability of microcrack formation [1].

Hence, the motive of this study is to evaluate the microcrack formation in apical third of root after retreatment.

\section{Materials and Methods}

50 Freshly extracted permanent mandibular premolar extracted for orthodontic reason, and having straight root and single canal confirmed by radiograph were collected for the study. The institutional ethical committee approved the study. The teeth were stored in $1 \%$ sodium hypochlorite to eliminate organic debris. They were then removed, washed under tap water and stored in 10\% Formalin solution for the disinfection of the teeth, till they were used for the purpose of the study.

Coronal access cavities were prepared with BR 41 round bur and canals were located with the help of a DG 16 probe. The patency was determined by 10 size and 15 size K-file placing into the canal until it was visible at the apical foramen. Whenever there is resistance with 10 size $\mathrm{K}$ file, Proglider were used. The working length was established with cusp tip as reference point to $0.5 \mathrm{~mm}$ short of anatomic apex. The coronal enlargement was done by one flare rotary file. Then the canals were instrumented with Protaper Next files coated with Glyde chelating paste size X1, X2. After the use of every file, $5.25 \%$ sodium hypochlorite is used for irrigation. $5.25 \%$ sodium hypochlorite and saline was used after complete preparation of the root canals. As a final irrigant, citric acid $40 \%$ was used.

Drying of each canal was obtained with paper points and endo aspirator. The gutta-percha cones were inserted into canals and verification of working length as well as fit at the apical third was done after the radiographic confirmation. Obturation was done using master gutta-percha cone with AH Plus sealer mixed according to the manufacturer's instruction by lateral condensation technique. Hand spreader and accessory cones were also used.

The gutta-percha was sheared off from the pulp chamber with a heated plugger at the level of canal orifice. The access cavity sealing were done with cotton pellet and temporary filling material (Cavit Espe Dental, Medizin, Germany).

The quality and apical extent of the root canal filling were assessed with radiograph and stored at $37^{\circ} \mathrm{C}$ in $100 \%$ relative humidity for 2 weeks to allow complete setting of the sealer.

\section{Retreatment}

The teeth were randomly assigned to 2 groups of 25 specimens each, and the canals were retreated with one of the following methods:

Group I-Protaper universal retreatment file (Dentsply Maillefer)

Protaper universal retreatment instruments were used to remove root filling by using D1, D2, D3 retreatment instruments. Protaper Universal retreatment file instruments were used in a brushing movements against the walls of the canal in a crown -down manner in cervical third by D1 (500 rpm and 2-3N/cm), in middle third by $\mathrm{D} 2$ (400rpm and $2-3 \mathrm{~N} / \mathrm{cm}$ ) and in apical third by D3 (400rpm and 1.5-2 N/cm) until reaching the working length as recommended by the manufacturer for removing sealer and guttapercha.

\section{Group II- Wave one gold file (Dentsply Maillefer)}

A primary reciprocating Waveone Gold primary file having a size 25 and a taper of 0.07 was used in a reciprocating mode ac- 
cording to manufacturer's instructions with controlled speed and torque with slow in-and-out pecking motion by an extended about $3 \mathrm{~mm}$ each time by the application of minimum apical pressure and brushing movements against canal walls. The cleaning of the flutes of the instruments were done after every pecks.

When the instrument did not carry any filling material after insertion and removal, considered as complete removal of the material. After the retreatment procedure, radiograph has taken for all roots for the verification of the removal of filling material by different removal techniques. Irrigation of root canals were done in between instrument changes using $5 \mathrm{ml}$ of $5.25 \% \mathrm{NaOCl}$ and smear layer will be removed by citric acid $40 \%$ and final irrigation with saline.

Tooth sectioning and microscopic examination

At the end, all the canals were washed and dried with absorbent paper points (Dentsply Maillefer). All the samples were sectioned at perpendicular to the long axis at $3 \mathrm{~mm}$ from the apex using a disc under water cooling. Two horizontal grooves were made on the buccolingual surfaces of each tooth and sectioned with chisel. 2.0\% glutaraldehyde aqueous solution was used to store specimens until the scanning by SEM. All the sections were then viewed under scanning electron microscope for the presence of microcracks and images were recorded.

\section{Statistical analysis}

The statistical analysis was done using statistical package of social sciences (SPSS) software, v.22, the comparison of microcracks incidence between groups was calculated by using Pearson Chisquare test. The difference will be considered as significant, when the $\mathrm{P}$ value is below 0.05 . The Odd's ratio and the Relative risk of the microcracks occurrence was calculated using the crosstabs function.

\section{Result}

The frequency (count) of microcracks among the samples in group I in which Protaper Universal retreatment file was used was found to be $68 \%$ (17), and microcracks were found to be absent in $32 \%$ (8). The frequency (count) of microcracks among the samples in group II in which Wave One Gold file was used was found to be $36 \%$ (9), and microcracks were found to be absent in 64\% (16). The data was outlined in table 1 to 3 and graph 1 to 4 and figure 1 and 2 .

\begin{tabular}{|l|c|c|c|c|c|}
\hline \multicolumn{1}{|c|}{ Group } & \multicolumn{2}{|c|}{ Microcrack } & Total \\
\cline { 2 - 5 } & Present & Percentage & Absent & Percentage & \\
\hline $\begin{array}{l}\text { Protaper } \\
\text { Universal } \\
\text { retreatment } \\
\text { file }\end{array}$ & 17 & $68 \%$ & 8 & $32 \%$ & 25 \\
\hline $\begin{array}{l}\text { Wave One } \\
\text { Gold file }\end{array}$ & 9 & $36 \%$ & 16 & $64 \%$ & 25 \\
\hline
\end{tabular}

Table 1: Comparison of Count and Percentage of microcracks in apical one third of root after retreatment in group I (Protaper Universal Retreatment File) and group II (Wave One Gold File).

\begin{tabular}{|l|l|l|l|}
\hline \multicolumn{4}{|c|}{ Risk Estimate } \\
\hline & \multirow{2}{*}{ Value } & \multicolumn{2}{|l|}{$\mathbf{9 5 \% \text { CI }}$} \\
\cline { 3 - 4 } & & Lower & Upper \\
\hline $\begin{array}{l}\text { Odds Ratio for Group (Protaper } \\
\text { Universal retreatment file / Wave } \\
\text { One Gold file) }\end{array}$ & 3.778 & 1.170 & 12.194 \\
\hline Relative Risk of Microcrack & 1.889 & 1.049 & 3.400 \\
\hline
\end{tabular}

Table 2: Relative risk of microcrack formation using Protaper Universal retreatment file and Wave One Gold file after retreatment in the apical one third of root.

\begin{tabular}{|c|l|l|l|l|}
\hline \multirow{2}{*}{$\begin{array}{c}\text { Micro- } \\
\text { crack }\end{array}$} & $\begin{array}{c}\text { Protaper } \\
\text { Universal } \\
\text { retreatment file }\end{array}$ & $\begin{array}{c}\text { Wave One } \\
\text { Gold file }\end{array}$ & $\begin{array}{c}\text { Chi-Square } \\
\text { Value } \\
{\left[\mathbf{X}^{2}\right]}\end{array}$ & P Value \\
\hline Present & $17(68 \%)$ & $9(36 \%)$ & 5.128 & $0.024^{*}$ \\
\hline Absent & $8(32 \%)$ & $16(64 \%)$ & & \\
\hline
\end{tabular}

Table 3: Incidence and comparison of Microcracks using both methods in apical one third of root after retreatment in group I (Protaper Universal Retreatment File) and group II (Wave One Gold File).

\section{Discussion}

Despite having high degree of success rate, sometimes root canal therapy may not lead to desire response and failure may occur. Reoccurrence of disease occurs because of continual bacteria in the canal which may be due to missed canal, improper cleaning, inappropriate filling or leakage [6]. When root canal therapy fails then, the treatment options are conventional retreatment or peri- 


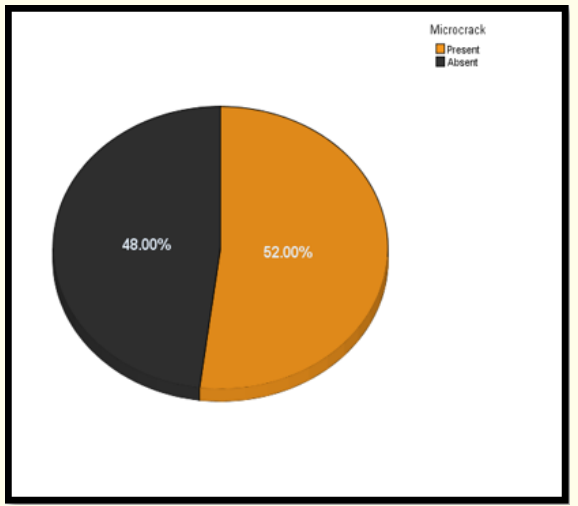

Graph 1: Pie chart showing the percentage of microcracks in the apical one third of root after retreatment in group I (Protaper Universal Retreatment File) and group II (Wave One Gold File).

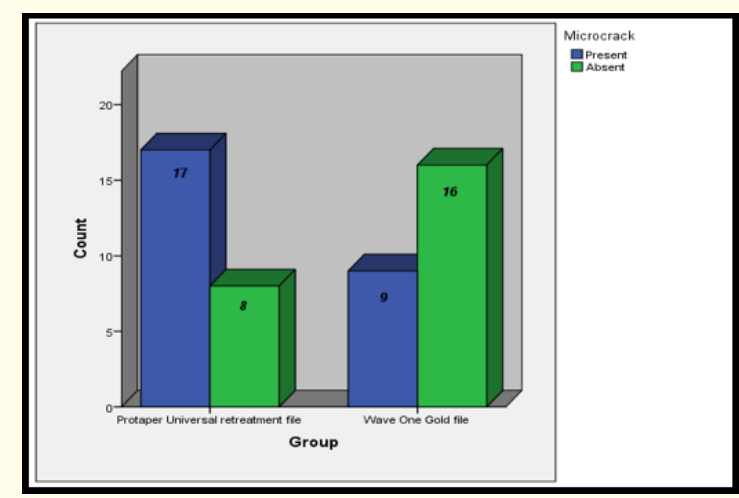

Graph 2: Bar chart showing the comparison of incidence of microcracks in apical one third of root after retreatment in group I (Protaper Universal Retreatment File) and group II (Wave One Gold File).

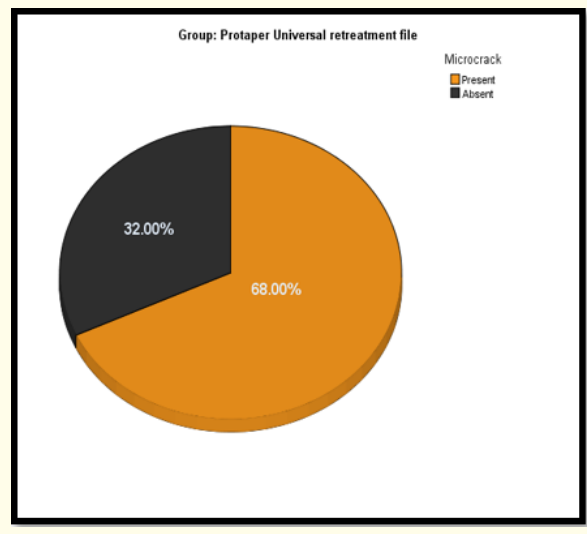

Graph 3: Pie chart showing the percentage of microcracks in the in apical one third of root after retreatment in group I (Protaper Universal Retreatment File).

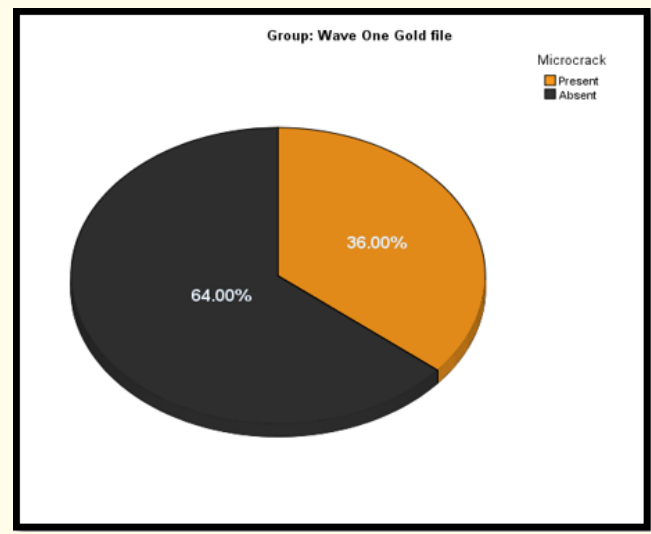

Graph 4: Pie chart showing the percentage of microcracks in the apical one third of root after retreatment in group II (Wave One Gold File).

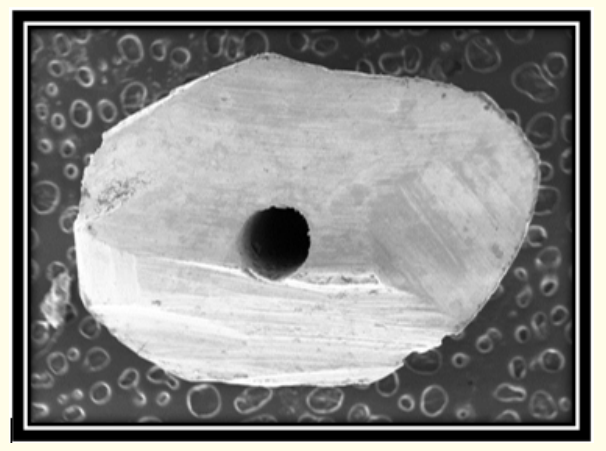

Figure 1: SEM image demonstrating no microcracks formation after retreatment.

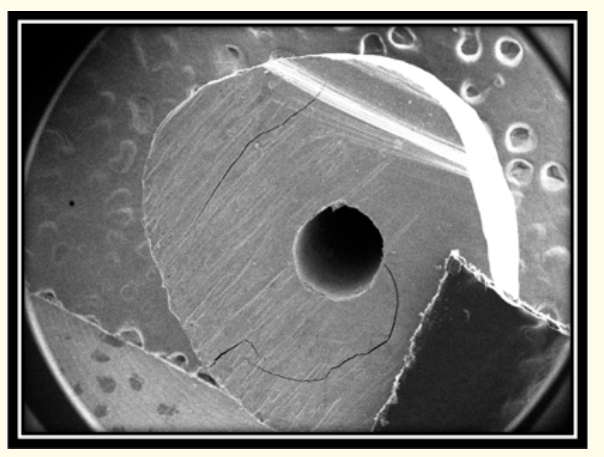

Figure 2: SEM image demostrating microcrack formation after retreatment.

radicular surgery. Diagnosis of unsuccessful endodontic treatment is done by clinical signs and symptoms and radiographic findings of tooth [5]. Properly and without damaging the remaining tooth 
structure, the filling material should be removed from canal for nonsurgical retreatment [6].

Generally, hand endodontic files made up of stainless steel were used for root canal treatment. Recently, new designs and methods of root canal treatment have emerged as there is development in the NiTi rotary instruments. Retreatment systems have several advantages, including reduced working time and self-centering ability. But the major drawback associated with rotary nickel instrumentation is the incidence of dentinal defects which further leads to vertical root fracture (VRF). Another problem with nickel titanium instrument is instrument separation. Cyclic fatigue and torsional fatigue are the main causative factors for instrument separation. Dentinal defects like microcracks may be formed due to the stress concentration that arise with the contact of dentin and endodontic instruments. These microcracks are most likely to develop into vertical root fracture (VRF), by the repeated tension through occlusal forces. Studies shows that VRF's are possibly developed from the minute dentinal defects instead of the forces while preparing and obturating the canal [7].

In our study, fifty mandibular premolars were used; the teeth were divided in two groups: In group I - Retreatment was done by using Protaper Universal Retreatment Files and in group II - Retreatment was done by using Wave One Gold Files.

Microcracks were evaluated after using above rotary retreatment files. Microcracks are evaluated by different methods. The studies done by Liu., et al. in year 2013; Yoldas., et al. in year 2012; Barreto., et al. in year 2012; Bier., et al. in year 2009; used digital cameras of magnification of 10,12,20,40 for viewing the microcrack. Though, introduction of scanning electron microscope (SEM) has proved to be beneficial method, as instrumentation and instrumentation techniques can be compared by estimating the potential of endodontic procedures to remove the bacteria and debris from canals. High resolution images can be obtained through SEM which can be used to check the effect of preparation on dentin, cleaning capability of different instruments and emergence of dentinal defects in endodontic treatment. Hence, the microcracks were evaluated using scanning electron microscope in our study [8].

All the samples were sectioned at $3 \mathrm{~mm}$ from the apex perpendicular to the long axis of the tooth using a disc under water cooling. All the sections were scanned under scanning electron microscope and the images were recorded. The scanned images were evaluated for dentinal defects as given by Shemesh., et al. (2009).
A root without any craze lines or microcracks at external surface of root or the internal root canal wall is given as "No defect - Absent".

A root dentin having all other lines like microcracks, fractures, a partial crack or craze lines is given as "Defects - Present" [8].

In our study, in group I - when Protaper Universal retreatment files were used for retreatment the results found to have cracks in $68 \%$ samples used, which is shown in graph 3.

The results obtained in our study is comparable with the study done by Topcuoglu H. in 2014, in his study Protaper universal retreatment files were compared to other retreatment rotary files, he concluded that the protaper universal retreatment files caused more crack initiation and propagation after retreatment in apical dentin [1].

Our results are also in accordance with the study done by Taha Ozyurek in 2017, when he used Protaper Universal Retreatment File for retreatment incidence of apical crack formation and propagation during retreatment causes more crack formation when compared with other nickel-titanium instruments he used in his study [3], by Jain A., et al in 2019 [9,10].

A significant difference was found in between group II and group I. The chisquare value was found to be 5.128 with $p$ value of 0.024 which is shown in table 3 .

The relative risk of the microcracks was found to be 1.889, which states that the microcracks were more likely occur using Protaper universal retreatment file, than Wave One Gold file. The Odd's ratio was found to be 3.778 , which states that the removal of root canal filling material by Protaper universal retreatment file is 3.778 times more prone for occurrence of microcracks in the apical third of the root, when compared to the Wave One Gold File (Table 2).

The results obtained in our study shows significant difference between group II- wave one gold files and group I- Protaper universal retreatment files.

As both the NiTi retreatment files works with two different types of movements- continuous rotating full sequence and reciprocating, hence there was difference in results obtained between both the groups. No previous studies till date have evaluated the formation of cracks when wave one gold file is used in retreatment. 
In our study, the results in the group II were significant from group I. The reasons could be as, Wave One Gold file works on reciprocation movement. It reduces the stresses by counter clockwise (Cutting action) and clockwise (release of instrument) movements on instrument. Reciprocating movement asserts to imitate manual movement and lowers the numerous risks related with continuous rotating file systems [11].

They are designed with reverse cutting helix, engage and cut dentin in a 150 degree counter-clockwise (CCW) direction, and when it has a chance to taper lock before that it disengages 30 degree in a clockwise (CW) direction [11].

It produces less tension on the walls due to the flexibility which is attained by the design and heat treatment of NiTi alloys and have parallelogram cross section having two 85 degree cutting edges and alternating with off-centered cross section having one cutting edge which contacts the canal wall [11].

A more conservative shaped canal with maximum preservation of tooth structure as it has variable and reducing tapers (D1 - 7\%, D8 - 6\%, D16 - 3\%) with active cutting length of $16 \mathrm{~mm}$. The reduction in mass of the centre of the tip and improved penetration into the canal is obtained due to the ogival, roundly tapered and semiactive tip [11].

According to meta-analysis reciprocating instruments have proved to possess comparable efficiency to standard rotary files during endodontic retreatment. It generates fewer cracks than conventional multiple file rotary systems with pure rotational movement, this is in accordance with our study results [12].

Hence, our study group II is significantly different from group I. In group I when Protaper universal retreatment files were used for retreatment they work on rotation motion which causes torsion and flexion, which leads to stresses on instruments. It is used for removing root filling materials and comprises of 3 different instruments with different tapers and tip diameters to remove the filling material. It has a convex cross section design [13].

When the instruments with the same taper and cross-section were compared, PTUR files produced the maximum number of microcracks. This significant increase in microcracks formation is attributed by force of continuous rotation as well as constant torque created by NiTi rotary files.
On the contrary, Berutti., et al. in 2012 concluded that there is stress release associated with the reciprocating motion of Wave one gold before progression into the canal. Moreover, the time required with waveone gold is less than the protaper rotary files. This might also be responsible for greater number of microcracks [14].

With more complex apical anatomy, there is a higher compaction and penetration of obturating materials which could be another reason leading to stresses. In addition, Varied tip sizes and tapers among the instruments used in initial stage preparation and canal retreatment instruments further explains stress formation [12]. Hence, the files have to exert more pressure and get stressed to remove the gutta percha from the apical area. Another factor in the present study that contributes to dentinal micracks formation beside different types of systems used, are operator skill, storage conditions and absence of periodontal cushioning effect in prepared samples [13].

\section{Conclusion}

Within the limitations this invitro study concludes,

1. Result showed significant statistical difference among the groups of retreatment file systems(Protaper Universal Retreatment File system and Wave One Gold file system).

2. Protaper Universal Retreatment File group produced significantly increased percentage of incidence and propagation of dentinal microcracks as compared to Wave One Gold file group.

3. Root canal retreatment procedures generate stresses that can produce and propagate cracks from within the root canal.

4. The reciprocating motion provided better success rate than the continuous rotation motion.

\section{Conflict of Interest}

The authors deny any conflicts of interest.

\section{Bibliography}

1. Topcuoglu H., et al. "Incidence of apical crack initiation and propagation during the removal of root canal filling material with Protaper and Mtwo rotary Nickel-Titanium retreatment instruments and hand files". The Journal of Endodontics 40.7 (2014): 1009-1012. 
2. Mehmet $\mathrm{C}$ and Taha 0. "Effect of different nickel-titanium rotary files on dentinal crack formation during retreatment procedure". Journal of Dental Research, Dental Clinics, Dental Prospects 11.5 (2017): 90-95.

3. Ozyurek T., et al. "Incidence of apical crack formation and propagation during removal of root canal filling materials with different engine driven nickel-titanium instruments". Restorative Dentistry and Endodontics 42.4 (2017): 332-341.

4. Ismail D., et al. "Effect of the size of the apical enlargement with rotary instruments, single-cone filling, post space preparation with drills, fiber post removal, and root canal filling removal on apical crack Initiation and propagation". The Journal of Endodontics 41.2 (2015): 253-256.

5. Tomar A., et al. "To compare the efficacy of different file systems to remove filling material during root canal treatment utilizing stereomicroscope: An in vitro study". International Journal of Applied Dental Sciences 4.2 (2018): 154-157.

6. Topcuoglu H., et al. "Effects of Mtwo, R-Endo, and D-RaCe retreatment instruments on the incidence of dentinal defects during the removal of root canal filling material". The Journal of Endodontics 40.2 (2014): 266-270.

7. Marcely C., et al. "Effects of Reciproc, ProTaper Next and WaveOne Gold on Root Canal Walls: A Stereomicroscope Analysis". Iranian Endodontic Journal 13.2 (2018): 228-233.

8. Cicek E., et al. "Evaluation of microcrack formation in root canals after instrumentation with different NiTi rotary file systems: A scanning electron microscopy study". Wiley Online Library Scanning 37.1 (2015): 49-53.

9. Jain A., et al. "Effect of root canal preparation, obturation, and retreatment on the induction of dentinal microcracks: A microcomputed tomography study". Journal of Conservative Dentistry 21 (2018): 521-25.

10. Sukul S., et al. "Incidences of Root Microcracks Caused by Hand and Rotary File System at Different Lengths". International Healthcare Research Journal 3.9 (2019): 283-289.

11. Webber J. "Shaping canals with confidence: WaveOne GOLD single-file reciprocating system". International Dentistry - African Edition 6.3 (2016): 6-17.
12. Frater M., et al. "The potential effect of instrumentation with different nickel titanium rotary systems on dentinal crack formation-An in vitro study". PLoS ONE 15.9 (2020): e0238790.

13. Prashant M., et al. "Comparison of incidence of dentinal defects after root canal preparation with continuous rotation and reciprocating instrumentation". Singapore Dental Journal 36 (2015): 29-33.

14. V Ashwinkumar., et al. "Effect of reciprocating file motion on microcrack formation in root canals: an SEM study". International Endodontic Journal 47.7 (2014): 622-627.

\section{Assets from publication with us}

- Prompt Acknowledgement after receiving the article

- Thorough Double blinded peer review

- Rapid Publication

- Issue of Publication Certificate

- High visibility of your Published work

Website: www.actascientific.com/

Submit Article: www.actascientific.com/submission.php

Email us: editor@actascientific.com

Contact us: +919182824667 